\title{
Treatment of carcinoma of the anal canal at Groote Schuur Hospital
}

\author{
B Robertson, L Shepherd, R P Abratt, A Hunter, P Goldberg
}

Objectives. Chemoradiation is the treatment of choice for squamous carcinoma of the anal canal, resulting in the same local control rates as surgery but with the advantage of organ function preservation. We aimed to review all cases of anal canal carcinoma treated at Groote Schuur Hospital between 2000 and 2004 and to assess treatment outcome.

Methods. The records for 31 patients presenting during this period were reviewed. Patient and tumour characteristics were recorded. Twenty-six patients were treated with chemoradiation. Local failurefree, colostomy-free and overall survival were calculated using the Kaplan-Meier method.
Results. Compared with the literature, the median patient age was younger and the stage was more advanced in this study. The complete response rate for all stages with chemoradiation was $80 \%$. The local failure-free survival at 5 years was $60.7 \%$. Colostomy-free and overall survival at 5 years were $59.2 \%$ and $65.6 \%$, respectively.

Conclusions. The patients presented with locally advanced disease. Chemoradiation is effective treatment for this group of patients and the majority avoid a permanent colostomy as they preserve anal sphincter function.

S Afr Med J 2012;102(6):558-561.
Patients with carcinoma of the anal canal may be treated with combination of radiation and chemotherapy to avoid the need for surgical resection of the anus and a permanent colostomy.

The disease is rare and accounts for approximately $1.8 \%$ of cancers of the gastrointestinal system in the USA. ${ }^{1}$ It comprises $4 \%$ of the total number of cancers of the gastrointestinal tract seen at Groote Schuur Hospital (GSH). Aetiological factors include human papillomavirus (HPV) infections, particularly type 16, immunosuppression, chronic inflammation and smoking.

The tumour arises in the anal transition zone and the majority are squamous carcinomas. Only $5-10 \%$ of patients present with metastatic disease. Before the 1970s - 1980s this disease was treated surgically with abdominoperineal resection (APR) resulting in a permanent colostomy. Recurrence rates were about $40 \%$ and the majority of relapses were locoregional. ${ }^{2}$ In 1984 the results on over 100 patients treated with chemoradiation were published by Nigro. ${ }^{3}$ There was a complete pathological response rate of $93 \%$. Results of subsequent trials have led to the recommendation that standard treatment for carcinoma of the anal canal is radiation to a dose of 45 - 50 Gy with a combination of 5 fluorouracil (5FU) and mitomycin $\mathrm{C}$ chemotherapy. ${ }^{4-8}$ APR is generally reserved for salvage treatment. Local excision may be considered for tumour stage 1 (T1) lesions.

The aim of this paper was to review the characteristics of patients presenting with anal canal carcinoma at GSH. We also reviewed the treatment and compared our local control, colostomy-free and overall survival rates for chemoradiation with those in the literature.

Department of Radiation Oncology, Groote Schuur Hospital and University of Cape Town

B Robertson, MB ChB, FC Rad Onc

L Shepherd, $\mathrm{MB} \mathrm{ChB}, \mathrm{DCH}$

R P Abratt, MB ChB, FC Rad Onc, MMed Rad T

A Hunter, $\mathrm{PhD}$

Department of Surgery, Groote Schuur Hospital and University of Cape Town P Goldberg, MB ChB, FCS, MMed

\section{Patients and methods \\ Patients and staging}

The records for all patients presenting with carcinoma of the anal canal between January 2000 and December 2004 were reviewed. The age, gender, race, stage and HIV status were recorded. Staging investigations included chest X-ray and ultrasound or computed tomography (CT) scan of the abdomen. Full blood count and serum chemistry were requested. Patients were staged according to the 1985 Union for International Cancer Control (UICC) classification. All patients were reviewed at a multidisciplinary team meeting for a management decision.

\section{Treatment}

For patients treated with chemoradiation, the radiation dose was either $42.00 \mathrm{~Gy}$ in 20 fractions or $44.20 \mathrm{~Gy}$ in 20 fractions (after June 2003) to a central dose, 4 fractions weekly using 60 -Cobalt. Anterior and posterior fields to the pelvis were based on bony landmarks as well as tumour extent. The superior border of the radiation field was placed at the lower border of the sacro-iliac joint or the upper border of the acetabulum. The inferior border was placed $2 \mathrm{~cm}$ below the anal verge or $2 \mathrm{~cm}$ below visible tumour if the tumour protruded from the anal canal. For patients with no inguinal node involvement the lateral border was $1 \mathrm{~cm}$ lateral to the widest brim of the pelvic side walls. In patients with involved inguinal nodes the lateral border was placed 2 $\mathrm{cm}$ lateral to the palpable nodes. Patients without stomas were treated prone. The chemotherapy regimen was mitomycin C $12 \mathrm{mg} / \mathrm{m}^{2}$ on day 1 and $5 \mathrm{FU} 1000 \mathrm{mg} / \mathrm{m}^{2}$ continuous infusion on days $1-4$, i.e. with the first 4 fractions, and $5 \mathrm{FU} 1000 \mathrm{mg} / \mathrm{m}^{2}$ with the last 4 fractions.

All patients were reviewed 6 weeks after completing treatment. If the size of the primary tumour had decreased by more than $50 \%$ a further dose of $15.00 \mathrm{~Gy}$ in 6 fractions was given to the perineum. Patients with less than $50 \%$ response proceeded to surgery. The response to treatment for patients receiving chemoradiation was assessed clinically at the completion of treatment. Overall survival, local control and colostomyfree survival were calculated using the Kaplan-Meier method. The ethical conduct of the study was acknowledged by the University of Cape Town Faculty of Health Sciences Research Ethics Committee.

\section{Results \\ Patient characteristics}

Thirty-one patients were included in the analysis. Table 1 illustrates the patient and tumour characteristics. The median age was 56 years (range 18 - 87). There were 15 female patients and 16 male patients. Of 
22 tested patients only 1 was positive for the human immunodeficiency virus (HIV). Locally advanced disease (T3, 4 tumours) was present in $67 \%$ of patients and $38 \%$ had palpable inguinal nodes. Metastatic disease was present in 1 patient. Only 1 patient had an adenocarcinoma; the remainder were squamous carcinomas.

Table 1. Patient and tumour characteristics

\begin{tabular}{ll}
\hline Characteristic & $\boldsymbol{N}(\%)$ \\
\hline Sex & $16(52)$ \\
Male & $15(48)$ \\
Female & \\
Age (years) & $56(18-87)$ \\
$\quad$ Median (range) & \\
Tumour stage & $1(3)$ \\
T1 & $10(32)$ \\
T2 & $17(55)$ \\
T3 & $3(10)$ \\
T4 & \\
Node stage & $19(62)$ \\
Inguinal nodes not involved & $12(38)$ \\
Involved inguinal nodes & \\
Metastatic stage & $30(97)$ \\
M0 & $1(3)$ \\
M1 & \\
Histology & $30(97)$ \\
Squamous carcinoma & $1(3)$ \\
Adenocarcinoma & $1(3)$ \\
HIV-positive & \\
&
\end{tabular}

\section{Treatment}

Table 2 shows the treatment summary. Chemoradiation was the primary treatment administered to 26 of the 31 patients. Of the remaining 5 patients, 2 underwent an APR as their primary treatment as they were not suitable for chemoradiation and 3 patients received no treatment (poor performance status in 2 and 1 failure to attend). A colostomy for obstructive symptoms or faecal incontinence was required in $23 \%$ ( 6 patients) prior to chemoradiation.

There was a less than $50 \%$ response at initial assessment after chemoradiation for 6 of the 26 patients. An APR was undertaken on 2 of these patients. Two patients were found to have no evidence of residual disease at the time of planned surgery and were followed up. Two patients did not receive further treatment after chemoradiation because of extensive disease not suitable for radiation boost or resection.

\section{Table 2. Patient treatment}

\begin{tabular}{ll}
\hline Modality & $\boldsymbol{N}(\%)$ \\
\hline Chemoradiation & $26(84)$ \\
Abdominoperineal resection & $2(6)$ \\
None & $3(10)$ \\
Stoma prior to chemoradiation & $7(30)$
\end{tabular}

\section{Toxicity}

Treatment was not completed in 7\% (2 patients) because of toxicity. Treatment interruptions for grade 2 or 3 toxicity were required in $38 \%$ (10 patients). The most common toxicity was radiation dermatitis. Details of toxicity are shown in Table 3. Treatment was interrupted until resolution to grade 1 toxicity.

\begin{tabular}{ll} 
Table 3. Treatment toxicity & \\
\hline Toxicity & $\boldsymbol{N}(\%)$ \\
\hline Acute & $7(27)$ \\
$\quad$ Radiation dermatitis & $1(3)$ \\
Thrombocytopenia & $1(3)$ \\
Neutropenia & $1(3)$ \\
Mucositis & $1(3)$ \\
Late & $1(3)$ \\
Anal stenosis & \\
Pain &
\end{tabular}

Late toxicity was observed in 2 patients treated with chemoradiation. One required a loop colostomy 3 years after completing treatment for anal stenosis causing faecal incontinence and 1 required an APR 2 years after chemoradiation for persistent anal pain. There was no histological evidence of recurrence.

\section{Treatment outcome}

The median follow-up time was 49 months. An initial local complete response to chemoradiation occurred in $80 \%$ of patients (21 of 26). There were relapses in $27 \%$ ( 7 patients). Details of treatment outcome are shown in Table 4 . Only 1 patient with local relapse was suitable for APR and remains clear.

\begin{tabular}{ll} 
Table 4. Treatment outcome & \\
\hline Parameter & $\boldsymbol{N}(\%)$ \\
\hline Initial complete response & $21(80)$ \\
No response & $4(15)$ \\
Lost to follow-up & $1(3)$ \\
Local relapse & $3(11)$ \\
Local and distant relapse & $2(7)$ \\
Distant relapse & $2(7)$
\end{tabular}

The Kaplan-Meier method was used to calculate the local failurefree, colostomy-free and overall survival rates for patients treated with chemoradiation. Local failure was defined as those patients not achieving a complete response to chemoradiation and those who relapsed locally. Our local failure-free survival at 5 years was $60.7 \%$. The colostomy-free survival was $59.2 \%$ at 5 years and the 5 -year overall survival was $65.6 \%$.

\section{Discussion}

The majority of articles on squamous carcinoma of the anal canal have been published in North America and Europe. Madden et al. ${ }^{9}$ published a retrospective review in 1981 on patients treated at GSH. Chemoradiation was not standard care at this time. A further paper discussing salvage surgery following chemoradiation was published in 1989 from the same institution. ${ }^{10}$ 
The purpose of this audit was to review the patient characteristics and outcome of treatment of anal canal carcinoma at GSH over a 4 -year period. We noted that there were more males than females. In general there is a slight female predominance for this disease. ${ }^{11}$ The reported median age at diagnosis in the USA is 58 years. ${ }^{11}$ Our median age was 56 years. In South Africa one would expect a high incidence of patients with HIV infection. Only 1 patient was HIV positive although the status of 9 patients was not known. The rate of metastasis at presentation is low and occurred in only 1 patient, which is consistent with the low rate of $5-10 \%$ of patients reported elsewhere. Advanced disease (T3, T4) at presentation was present in $67 \%$, which is higher than in the USA where $30 \%$ of patients present with T3 or T4 tumours. ${ }^{11}$ Inguinal nodes were present in 38\%. This is also much higher than the $20-25 \%$ of patients who presented with involved nodes reported from the USA. ${ }^{11}$

Chemoradiation was administered in $93 \%$ of patients. Grade 2 - 3 skin and haematological toxicity requiring a break in treatment occurred in $38 \%$ of patients. An initial complete response to treatment was observed in $80 \%$ of patients. However, $27 \%$ subsequently relapsed, the majority experiencing local relapse.

We compared the outcome of our patients treated with chemoradiation with that reported in the literature. The United Kingdom Co-ordinating Committee on Cancer Research (UKCCCR) and the European Organisation for Research and Treatment of Cancer (EORTC) used a similar regimen of mitomycin C, 5FU and 45.00 50.00 Gy split-course radiation in the chemotherapy plus radiation arms. ${ }^{3,4}$ The local control rate at 5 years for this regimen in the EORTC trial was $69 \%$ while the local failure-free rate for the UKCCCR trial at 5 years was $32 \%$. Our local control rate at 5 years was $60.7 \%$. The colostomy-free survival at 5 years in the UKCCCR trial was $47 \%$. Our colostomy-free survival was $59.2 \%$. Overall survival on these trials in the chemotherapy arms was $58 \%$ at 5 years. Our overall survival was $65.6 \%$ at 5 years. Our results are therefore comparable.

Subsequent trials have reported higher colostomy-free and overall survival figures. A recent phase III trial (ACT II), which prescribed radiation with no planned treatment gap and compared cisplatin with $5 \mathrm{FU}$, as well as the role of maintenance chemotherapy, reported a $4-5 \%$ colostomy rate and over $80 \%$ overall survival at 3 years. ${ }^{8}$ Locoregional control and overall survival are significantly affected by $\mathrm{T}$ and $\mathrm{N}$ stage. ${ }^{12,13}$ In the ACT II trial $44 \%$ of patients had T3, T4 disease and $62 \%$ had negative nodes. In our patients $65 \%$ had T3 and $\mathrm{T} 4$ disease and $38 \%$ had positive nodes. Although a different treatment regimen was used in ACT II, the local control and survival rates of our patients are probably related to advanced disease at presentation.

The major advantage of treatment with chemoradiation as opposed to surgery is organ preservation. Therefore colostomy-free survival is an important endpoint. In general the reason for performing a colostomy is APR for persistent disease or recurrence following treatment. However $23 \%$ of our patients required colostomies for faecal incontinence or obstruction prior to chemoradiation. Only 1 patient had an attempt at reconstruction but subsequently developed faecal incontinence and required replacement of the colostomy. It was not possible to reverse the colostomies in the remaining patients because of loss of sphincter function. Only 3 patients (11\%) required an APR for persistent disease or recurrence. Therefore the major contribution to our colostomy rate was locally advanced disease at presentation.

Our radiation techniques were simple with two-dimensional (2D) planning and treatment with 60 -Cobalt. $5 \mathrm{FU}$ and mitomycin $\mathrm{C}$ chemotherapy agents are readily available and affordable. Our results, even for patients with locally advanced disease, were comparable to the UKCCCR and EORTC trials. Therefore curative treatment of carcinoma of the anal canal is possible with limited resources.

There is evidence that $3 \mathrm{D}$ conformal radiation treatment (3DCRT) using a high-energy linear accelerator is associated with less skin toxicity. ${ }^{14}$ This enables patients to complete treatment without breaks, which may improve local control. ${ }^{15,16}$ We have changed our treatment protocol to a continuous regimen of $50.00 \mathrm{~Gy}$ in $2.00 \mathrm{~Gy}$ fractions using 3D conformal radiotherapy where possible.

On review of the presenting history of many of our patients, we established that there had been several consultations with primary care physicians prior to referral and there was often a delay in diagnosis. It was also noted that some patients had been reluctant to seek medical attention in spite of having significant symptoms.

\section{Conclusions}

Squamous carcinoma of the anal canal is a disease which can be cured with radiation and chemotherapy while maintaining bowel function. The outcome however is related to disease stage at presentation. The patients in this study presented with advanced disease. The local control and overall survival rates at 5 years of $60.7 \%$ and $65.6 \%$ respectively compare favourably with trials using a similar treatment regimen. Cure can be achieved with widely available radiation techniques and chemotherapy agents. Early detection of this disease will improve the outcome for these patients.

Acknowledgements. The authors acknowledge Dr C Geddes and Dr R Baigrie who were involved with the treatment of some of these patients.

\section{References}

1. Jemal A, Siegel R, Ward E, et al. Cancer Statistics CA. Cancer J Clin 2008;58(2):71-96. [http://dx.doi. org/10.3322/CA.2007.0010]

2. Boman BM, Moertel CG, O'Connell MJ, et al. Carcinoma of the anal canal: a clinical and pathologic study of 188 cases. Cancer 1984;54(1):114-125. [http://dx.doi.org/10.1002/1097-0142(19840701)]

3. Nigro ND. An evaluation of combined therapy for squamous cell cancer of the anal canal. Dis Col Rect 1984;27(12):763-766. [ http://dx.doi.org/10.1007/BF02553933]

4. UKCCCR Anal Cancer Trial Working Party. Epidermoid anal cancer: results from the UKCCCR randomised trial of radiotherapy alone versus radiotherapy, 5-fluorouracil, and mitomycin. Lance 1996;348:1049-1054. [http://dx.doi.org/10.1016/S0140-6736(96)03409-5]

5. Bartelink $\mathrm{H}$, Roelofsen $\mathrm{F}$ Eschwege $\mathrm{F}$ et al. Concomitant radiotherapy and chemotherapy is superior to radion randomized trial of the European Organization for Research and Treatment of Cancer Radiotherapy randomized trial of the

6. Flam M, John M, Pajak TF, et al. Role of mitomycin in combination with fluorouracil and radiotherapy and of salvage chemoradiation in the definitive nonsurgical treatment of epidermoid carcinoma of the anal canal: Results of a phase III randomized intergroup study. J Clin Oncol 1996;14(9):2527-2539.

7. Ajani JA, Winter KA, Gunderson LL, et al. Fluorouracil, mitomycin and radiotherapy vs fluorouracil, cisplatin, and radiotherapy for carcinoma of the anal canal. JAMA 2008;299(16):1914-1921. [http:// dx.doi.org/10.1001/jama.299.16.1914]

8. James R, Wan S, Glynne-Jones R, et al. A randomized trial of chemoradiation using mitomycin or cisplatin, with or without maintenance cisplatin/5FU in squamous cell carcinoma of the anus (ACT II). J Clin Oncol 2009;27(18S):797s (abstract LBA 4009).

Madden MV, Elliot MS, Botha JBC, et al. The management of anal carcinoma. Br J Surg 1981;68:287289. [http://dx.doi.org/10.1002/bjs.1800680420] [PMID:7225745]

10. Palmhert IJ, Goldberg PA, Geddes C, et al. Does failure of primary chemo-radiotherapy for squamous carcinoma of the anal canal predict death? S Afr J Surg 1998;36(3):106.

1. Bilimoria $\mathrm{KY}$ Bentrem $\mathrm{DJ}, \mathrm{Ko} \mathrm{CY}$ et al. Squamous cell carcinoma of the anal canal. Utilization and B outcomes of recommod treatint in the Uni Das.

Das for anal cancer. Int J Rad Oncol Biol Phys 2007;68(3):794-800. [http://dx.doi.org/10.1016/j. ijrobp. 2006.12.052]

3. Ajani JA, Winter KA, Gunderson LL, et al. US Intergroup anal carcinoma trial: tumor diameter predicts for colostomy. J Clin Oncol 2009;27(7):1116-1121.

14. Vuong T, Kopek N, Ducruet T, et al. Conformal therapy improves the therapeutic index of patients with anal canal cancer treated with combined chemotherapy and external beam radiotherapy. Int $J \mathrm{Rad}$ Oncol Biol Phys 2007;67(5):1394-1400. [http://dx.doi.org/10.1016/j.ijrobp.2006.11.038]

15. Graf R, Wust P, Hildebrandt B, et al. Impact of overall treatment time on local control of anal cancer treated with radiochemotherapy. Oncology 2003;65:14-22. [http://dx.doi.org/10.1159/000071200]

16. Ben-Josef E, Moughan J, Ajani JA, et al. Impact of overall treatment time on survival and local contro in patients with anal cancer: a pooled data analysis of radiation therapy oncology group trials 87-04 and 98-11. J Clin Oncol 2010;28(34):5061-5066.

Accepted 31 January 2012. 